\title{
Urogenital Myiasis in Wolaita Sodo, Southern Ethiopia: Case Report
}

\author{
Abraham Getachew Kelbore $^{1^{*}} \quad$ Efa Ambaw Bogino ${ }^{1} \quad$ Blen kassahun Dessu $^{2} \quad$ Teshome Tesfaye ${ }^{3,4}$ \\ 1.Departement of Dermatology,School of Medicine, College of health science and medicine, Wolaita sodo \\ University, Ethiopia \\ 2. Department of anesthesia, College of Health sciences and Medicine, wolaita Sodo University, Ethiopia \\ 3 .Center for Academic and Health policy, Tehran University of Medical Sciences, Tehran \\ 4. Kembata Tembaro Zone Health Department, Southern Ethiopia
}

\begin{abstract}
Background: Myiasis is the infestation of human beings with fly larvae which feed on hosts living or dead tissue. The diagnosis of this case made by presence of larvae in affected body parts. In tropical countries myiasis can present commonly but still there is no a report of Urogenital myiasis case presentation in the case of Ethiopia.

Case presentation: A 19 years old male patient presented to Dermatology clinic at wolaita sodo university teaching and referral hospital with complaining of an itchy and painful small red raised/nodular/a painful penile lesion on the urethral meatus and glans of penis and on physical examination shows well demarcated centrally punctuated nodular lesion over the glans of the penis and urethral meatus with serous exudates discharge from opening that had appeared on the lesion. He had been treated with antibiotics treated for suspected Furuncle before three weeks at early onset, but not improved. Then the patient was successfully managed by manual removal of larvae after white petrolatum Vaseline application and local anesthesia for twenty minutes and antibiotics therapy. Conclusion: Genital myiasis should be considered as differential diagnosis for nodular lesions on the gentalia, particularly in tropical countries where myiasis is more frequent, as in the case presented.
\end{abstract}

Keywords: Myiasis, Urogenital, wolaita sodo, Infestation

DOI: $10.7176 / \mathrm{JHMN} / 59-08$

\section{Background}

Myiasis is a diseases caused by the infestation of body tissue by the larvae of several fly species ${ }^{[1]}$. Regarding their life cycle there are several ways for the flies to transmit their larvae to people. Some flies like attach their eggs to mosquitoes to bite people. Their larvae then enter these bites. The larvae feed in sub epidermal cavity for 5- 10 weeks, breathing through the hole in the host's skin. Other flies larvae burrow in to skin, these flies larvae are known as screwworms. They can enter to skin through people's bare foot, when they walk through soil containing fly eggs or attach themselves to people's wet clothes and burrow in to skin. Some of flies again deposit their eggs on or near to a wound or sore, depositing egg in to sloughing dead tissue. Whereas pathophysiology of Myiasis is dependent on the type of fly involved ${ }^{[2]}$ Myiasis clinically can present in deferent forms like furuncular Myiasis, wound Myiasis and nasal Myiasis.

We report a very rare presentation of Urogenital myiasis and the need for prevention of such cases to reduce the secondary infection, economical and psychological burden on the patients.

\section{Case presentation}

A 19 years old male high school student, patient living in the urban area of wolaita sodo, presented to Dermatology clinic at wolaita sodo university teaching and referral hospital in Sodo town, Ethiopia, complaining of a painful penile lesion on the urethral meatus and glans of the penis. The lesion had started on three weeks previously as an itchy and painful small red raised/nodular/ on the glans of the penis. After a few days a patient reported having the blood stained serous exudates began to discharge from opening that had appeared on the lesion. He sought medical help at nearby private medium clinics as soon as the lesion appeared and treated for suspected Furuncle. The patient recalled that, shortly before appearance of his penile lesion, he had noticed more flies at home surrounding than usual and dressed moist cloth which was hung outside for drying. It was his practice to wear washed clothes without Ironing. He did not have sexual contact and trauma history.

Physical Examination of these genitalia revels that well demarcated erythematous area of $1.5 \times 1.3 \mathrm{~cm}$ adjust to urethra meatus (fig 1). Then lesion contains $2 \mathrm{~mm}$ diameter of orifice from which serohemeorhagic /exudates/ materials could orifice, it was possible to observe a larvae moving around inside to lesional cavity. And it was difficult to determine where here has one larvae or more on manipulation of the glans of the penis. There is no associated limadinophaty.

\footnotetext{
${ }^{1}$.Vilcinskas A. From traditional maggot therapy to modern biosurgery. In: Vilcinskas A, ed. Insect Biotechnology. Dordrecht: Springer, 2011:67-75

2. Giuseppe Gaido . Myiasis, kenya. Comm Dermatol J 2014; 10: 1-12
} 


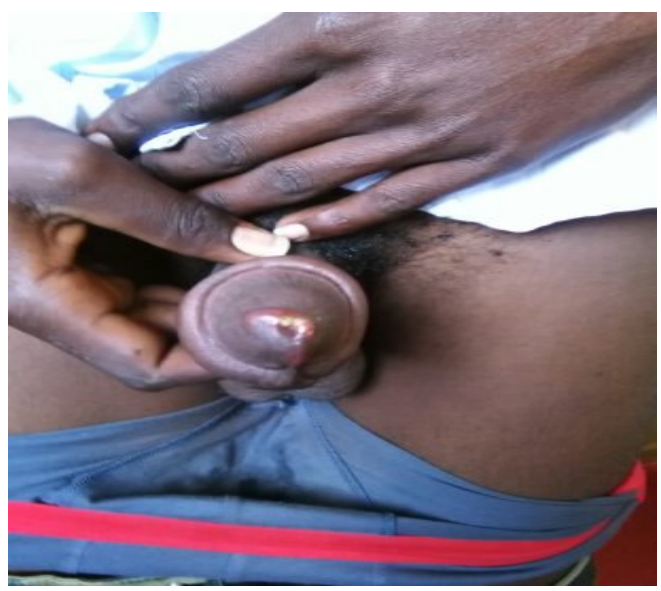

Fig 1Nodular lesion over glans penis and urethral meatus

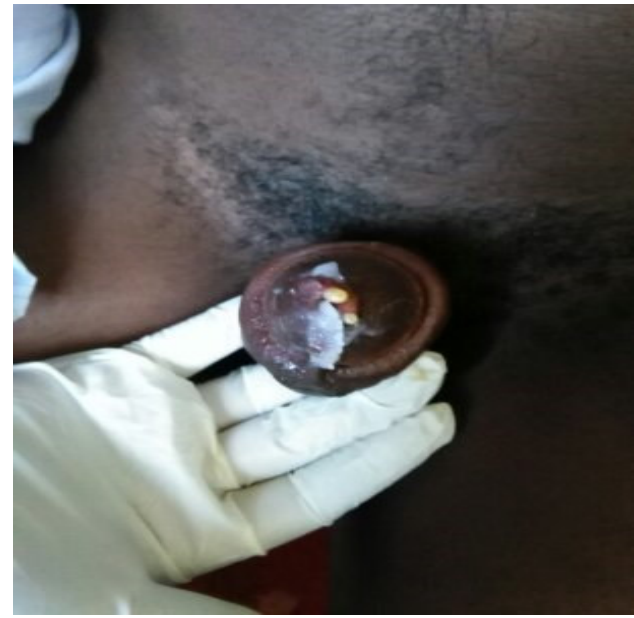

Fig 2 photo shows Glans penis and urethral meatus showing posterior aspect of the larvae with in lesional orfices

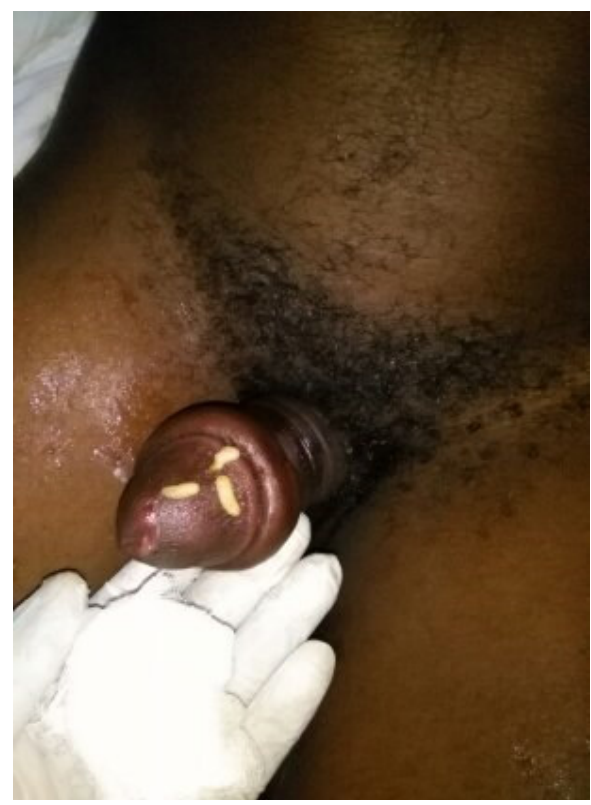

Fig 3 photo after removal of the larvae on the glans of the penis . 
After antisepsis with Iodine solution, the patient was given a local anesthesia of $2 \%$ lidocaine around the lesion's orifice, taking care to do not reach the larvae. The orifice was applied with White petrolatum Vaseline ointment for twenty minutes to make easier the larvae removal. (Fig 2)

Thereafter, by itself the larvae starts to emerge out with dorsal aspect and then a manual pressure were applied on the glands penis resulted in larvae expelling through the opened cavity. (Fig 3) After that, the cavity was cleaned with sterile $0.9 \%$ sodium chloride solution and iodopovidone. To prevent secondary infection, it was prescribed Cephalexin $500 \mathrm{mg}$ one tablet at every eight hours for seven days and the patient advice to take personal measures on how to prevent, like ironing washed clothes before wearing, left clothing's at list for three days after washed. The material removed (the larvae) was not sent for further identification species type due to absence of well equipped parasitological set up in the hospital.

\section{Discussion}

Our patient case report atypical site presentation of is Furuncular myiasis type , the most common manifestation of myiasis [Fig 1], produces typically boil-like lesions that may be painful, pruritic and tender. An erythematous papule, $1-3 \mathrm{~cm}$ in diameter and up to $1 \mathrm{~cm}$ in height, develops with 24 hours of infection. There may be the sensation of something moving under the skin. The lesion has a central punctum with serosanguinous discharge. Larvae rely on the central punctum to provide airflow to breathe, and bubbles may beseen exuding from the punctum. The primary reaction or secondary infection may produce lymphangitis and regional lymphadenopathy (fever, swollen glands, swollen extremities). If not removed, the larvae of most species will spontaneously emerge, leaving behind an exit wound ${ }^{[2,3,4]}$.

Diagnosis of myiasis is made by direct visualization of the larvae. A full blood count (Hemogram) may show leukocytosis and eosinophilia.

Treatment of myiasis typically involves direct surgical extraction of the larvae under local anesthesia. Secondline options involve suffocation techniques to encourage the larvae to migrate out of the skin through the use of petroleum jelly, and our case report patient was treated with option.

Third-line treatment with topical or systemic Ivermectin may be very helpful with orbital myiasis. Proper hygiene of wounds is very important when treating myiasis and is fundamental for prevention ${ }^{[2]}$ Genital myiasis should be considered as differential diagnosis for nodular lesions on the genitalia, particularly in tropical countries where myiasis is more frequent ${ }^{[5,6]}$.

Health education is paramount for the prevention of myiasis. In this connection the patient is advised to cease wearing wet clothes after washing. And told to avoid drying clothes on the lawn. The knowledge of the disease, its management and prevention is necessary for all health workers in tropical Africa, especially in Ethiopia.

Acknowledgement We thank the patient for willingness to give the lesion photos and college of health science and medicine for permission of this case report publication.

Funding The authors state that there was no funding.

Ethics approval and Consent: Ethical approval letter were secured from college of health science and medicine and Written informed consent was obtained from the patient for publication of this case report and the images.

\section{Authors' Contribution}

Abraham G Initiated case report, performed data collection, photography of clinical images, and was the main contributor in drafting and revising the manuscript. Efa A, Teshome T and Blen K were key contributors to the revisions of the manuscript. All authors read and approved the final manuscript.

Conflict of interests: No conflict of interests is declared.

\footnotetext{
${ }^{3}$.Lee EJK, Robinson F. Furuncular myiasis of the face caused by larva of the Tumbu fly (Cordylobia anthropophaga). Eye 2007; 21(2):266-268. [http://dx.doi.org/10.1038/sj.eye.6702507]

${ }^{4}$. Mashhood AA.Furuncular myiasis of the breast caused by the larvae of tumbufly. JColl physiciasins surg pak 2003;13:19-7

5 .Hannam P, Khairnar K, Downey J, Povis J, Ralevski F, Pillai DR . Cutaneous myiasis in traveler returning from Ethiopia. Emerg Infect Dis 2011; 17: 2385-2386.

6 . MRL. Passos. et al. penile myiasis:case report .sex transm infec 2004;80:183-184
} 